\title{
Convenient estimators for the panel probit model: Further results
}

\author{
William Greene $\mathrm{a}^{\mathrm{a}^{*}}$ \\ ${ }^{a}$ Department of Economics, Stern School of Business, New York University, \\ 44 West $4^{\text {th }}$ St., New York, NY, 10012, USA.
}

March 26, 2002

\begin{abstract}
Bertschek and Lechner (1998) propose several variants of a GMM estimator based on the period specific regression functions for the panel probit model. The analysis is motivated by the complexity of maximum likelihood estimation and the possibly excessive amount of time involved in maximum simulated likelihood estimation. But, for applications of the size considered in their study, full likelihood estimation is actually straightforward, and resort to GMM estimation for convenience is unnecessary. In this note, we reconsider maximum likelihood based estimation of their panel probit model then examine some extensions which can exploit the heterogeneity contained in their panel data set. Empirical results are obtained using the data set employed in the earlier study.
\end{abstract}

JEL classification: $\mathrm{C} 14 ; \mathrm{C} 23 ; \mathrm{C} 25$

Keywords: Panel probit model; Multivariate probit; GMM; Simulated likelihood; Latent class; Marginal effects

\footnotetext{
*E-mail: wgreene@stern.nyu.edu. Helpful comments and suggestions by Michael Lechner are gratefully acknowledged. Any remaining errors are the responsibility of the author.
} 


\section{Introduction}

Bertschek and Lechner (1998) (henceforth BL) propose a set of "convenient" GMM estimators for the binomial probit model based on panel data. Their GMM approach is motivated by the difficulty of computation of the log likelihood function for a fully unrestricted model with freely correlated disturbances when there are $T>2$ time periods. Because of the need to use a simulation based estimator, the primary obstacle is the actual amount of computation. They also argue that estimation of the disturbance covariance matrix, which involves $T(T-1) / 2$ free parameters, is unattractive because of the large size of the estimation problem. This note will compare the full likelihood based estimator to their GMM estimator. Speeds of computation have improved to the point that for a problem of the size of their application, the issue of computation time that was a focus of the earlier article is a minor consideration. More importantly, estimation of the full covariance matrix is revealing about the structure of the model in a way that would not be evident from their GMM approach. We will also examine two extensions of the panel probit model that were not considered by BL. The panel data set provides information about individual heterogeneity that is not exploited by the GMM estimator. We will reexamine their data set in the context of a random parameters model and a latent class model, both of which provide a means of examining the individual heterogeneity. The random parameters model has been widely used in the discrete choice (multinomial logit) framework, but has seen very limited application in the probit model, and none in the form considered here. The latent class model has been used almost exclusively to study count data. It appears not to have been employed in the analysis of binary choice data.

Section 2 of this paper describes the maximum likelihood and GMM panel data parameter estimators,. Section 3 discusses the common effects models and two extensions of the probit model, random parameters and latent classes. The application is described in Section 4. ${ }^{1}$ Some conclusions are drawn in Section 5. The random parameters model is estimated using a quasi Monte Carlo simulation method that has not

\footnotetext{
${ }^{1}$ The authors of the earlier study have generously allowed us to reuse their data for this analysis. Their assistance is gratefully acknowledged.
} 
yet seen wide use in econometrics. Some notes on this technique are provided in the appendix.

\section{Estimation of the panel probit model}

The panel probit model is

$$
\begin{aligned}
& y_{i t}^{*}=\mathbf{x}_{i t}^{\prime} \beta^{0}+\varepsilon_{i t}, t=1, \ldots, T, i=1, \ldots, N, \\
& y_{i t}=\mathbf{1}\left(y_{i t}^{*}>0\right) .
\end{aligned}
$$

The data consist of $N$ observations on $\mathbf{Z}_{i}=\left(\mathbf{y}_{i}, \mathbf{X}_{i}\right)$ where $\mathbf{y}_{i}=\left(y_{i 1}, y_{i 2}, \ldots, y_{i T}\right)^{\prime}$ and the $T$ rows of the $T \times K$ matrix $\mathbf{X}_{i}$ are $\mathbf{x}_{i t}^{\prime}, t=1, \ldots, T$. The disturbances are freely correlated across periods, with $T \times T$ positive definite covariance matrix $\Sigma$. The typical element of $\Sigma$ is denoted $\sigma_{t s}$. The standard deviations, $\sqrt{\sigma_{t t}}$ are denoted $\sigma_{t}$. The data on $\mathbf{x}_{i t}$ are assumed throughout to be strictly exogenous, which implies that $\operatorname{Cov}\left[\mathbf{x}_{i t}, \varepsilon_{j s}\right]=\mathbf{0}$ across all individuals $i$ and $j$ and all periods $t$ and $s$. This rules out state persistence, or the presence of lagged dependent variables in (1). ${ }^{2}$

The model in (1) is a special case of the $M$ equation multivariate probit model

$$
\begin{aligned}
& y_{i m}^{*}=\mathbf{x}_{i m}^{\prime} \boldsymbol{\beta}_{m}^{0}+\varepsilon_{i m}, m=1, \ldots, M, i=1, \ldots, N, \\
& y_{i m}=\mathbf{1}\left(y_{i m}^{*}>0\right)
\end{aligned}
$$

in which the parameter vectors are identical across equations. ${ }^{3}$ The observed data contain no information on the scaling of $y^{*}$, so the diagonal elements of $\Sigma, \sigma_{t t}$, are usually normalized to one. In the model in (1), however, normalization of all the diagonal elements is unnecessary because the slope vector is time invariant; $T$-1 ratios, $\sigma_{1} / \sigma_{t}$ are identified. As noted by BL, then, only one main diagonal element of $\Sigma, \sigma_{11}$, is normalized to one for identification purposes $-\beta^{0}$ is identified only "up to scale." Since the scaling may be different across periods, an equivalent formulation of (1) which embodies the convenient normalization $\sigma_{t t}=1$ while not restricting the variance parameters is obtained by scaling the coefficient vectors, instead;

\footnotetext{
${ }^{2}$ See Heckman (1981) and Woolridge (1995) for discussion.

${ }^{3}$ This is an $M$-variate extension of the bivariate probit model used, e.g., in Rubinfeld (1983), Boyes, Hoffman and Low (1989), Greene (1992), Burnett (1997) or Greene (1998).]
} 


$$
\begin{aligned}
& y_{i t}^{*}=\mathbf{x}_{i t}^{\prime} \beta^{0} \theta_{t}^{0}+\varepsilon_{i t}, t=1, \ldots, T, i=1, \ldots, N, \\
& y_{i t}=\mathbf{1}\left(y_{i t}^{*}>0\right) .
\end{aligned}
$$

where $\theta_{t}^{0}=\sigma_{1} / \sigma_{t}$. The normalization imposed by BL, $\sigma_{11}=\sigma_{t t}=\sigma_{T T}$ - see their footnote 2 on page 332 - is therefore restrictive. ${ }^{4}$ It is equivalent to an assumption that $\theta_{t}^{0}=1, t=$ $2, \ldots, T$, or that the disturbances are homoscedastic through time. While the assumption is substantive, for the data in their application, it does appear to be reasonable. The scale parameters $\theta_{t}^{0}$ are identified (estimable), so in principle, the restriction could be tested in a given application. ${ }^{5}$

Maximum likelihood estimates of the parameters in (3) with the homoscedasticity assumption are obtained by maximizing the log likelihood function,

$$
\log L=\sum_{i=1}^{N} \log \Phi^{(T)}\left(a_{i 1}, a_{i 2}, \ldots, a_{i T} \mid \Sigma^{*}\right)
$$

with respect to the unknown elements in $\beta$ and $\Sigma$ where, $\sigma_{t t}=1, a_{i t}=\left(2 y_{i t}-1\right) \mathbf{x}_{i t}{ }^{\prime} \beta, \sigma_{t s}{ }^{*}$ $=\left(2 y_{i t}-1\right)\left(2 y_{i s}-1\right) \sigma_{t s}, t \neq s$, and $\Phi^{(T)}$ denotes the CDF of the $T$-variate normal distribution. (The restriction, $\theta_{t}^{0}=1$ has already been imposed.) When $T$ exceeds two, computation of this function requires a multidimensional integration which can only be approximated. This can be done by simulation methods. The GHK simulator was used here. ${ }^{6}$ BL note three obstacles to estimation of this model: first, the amount of computation seems to be excessive ("prohibitively high"), which for their application is actually not the case - see the application below; second, global concavity may be a problem; and third, there are a large number of nuisance parameters in the model. BL's analysis produced several strategies for estimation designed to circumvent estimation of the off diagonal elements of $\Sigma$. But, in the application below, the estimated elements of $\Sigma$ are actually revealing in terms of suggesting a useful simplification of the model.

\footnotetext{
${ }^{4} \mathrm{BL}$ do consider an equivalent but less convenient normalization in their footnote 2 (p. 332). We will retain this normalization in what follows.

${ }^{5}$ This would require a full information maximum likelihood estimator, such as the one suggested here. BL's development was focused primarily on GMM estimators which obviate the calculation of $\Sigma$.

${ }^{6}$ See Hajivassiliou and Ruud (1994), Hajivassiliou et al., (1996), Geweke et al. (1994, 1997) and Geweke (1997).
} 
BL suggest a set of GMM estimators based on the orthogonality conditions implied by the single equation conditional mean functions;

$$
E\left\{\left[y_{i t}-\Phi\left(\mathbf{x}_{i t}^{\prime} \boldsymbol{\beta}\right)\right] \mid \mathbf{A}\left(\mathbf{X}_{i}\right)\right\}=0
$$

where $\Phi$ denotes the CDF of the univariate normal distribution and $\mathbf{A}\left(\mathbf{X}_{\mathrm{i}}\right)$ is a $P \times T$ matrix of instrumental variables constructed from the exogenous data for individual $i$. The orthogonality conditions are

$$
E\left[\mathbf{A}\left(\mathbf{X}_{i}\right)\left(\begin{array}{c}
{\left[y_{i 1}-\Phi\left(\mathbf{x}_{i 1}^{\prime} \boldsymbol{\beta}\right)\right]} \\
{\left[y_{i 2}-\Phi\left(\mathbf{x}_{i 2}^{\prime} \boldsymbol{\beta}\right)\right]} \\
\vdots \\
{\left[y_{i T}-\Phi\left(\mathbf{x}_{i T}^{\prime} \boldsymbol{\beta}\right)\right]}
\end{array}\right)\right]=\mathbf{0} .
$$

Using only the raw data as $\mathbf{A}\left(\mathbf{X}_{i}\right)$, strict exogeneity of the regressors in every period [see Woolridge (1995)] would provide $T K$ moment equations of the form $\mathrm{E}\left[\mathbf{x}_{i t} u_{i s}\right]=\mathbf{0}$ for each period, or a total of $T^{2} K$ moment equations altogether for estimation of $K$ parameters in $\beta$. The full set of such orthogonality conditions would be $E\left[\left(\mathbf{I}_{T} \otimes \mathbf{x}_{i}\right) \mathbf{u}_{i}\right]=\mathbf{0}$ where $\mathbf{x}_{i}=$ $\left[\mathbf{x}_{i 1}^{\prime}, \ldots, \mathbf{x}_{i T}^{\prime}\right]^{\prime}$. For our application, (6) produces 200 orthogonality conditions for the estimation of the 8 parameters. ${ }^{7}$ The empirical counterpart to the left hand side of (6) is

$$
\mathbf{g}_{N}(\boldsymbol{\beta})=\frac{1}{N} \sum_{i=1}^{N}\left[\mathbf{A}\left(\mathbf{X}_{i}\right)\left(\begin{array}{c}
{\left[y_{i 1}-\Phi\left(\mathbf{x}_{i 1}^{\prime} \boldsymbol{\beta}\right)\right]} \\
{\left[y_{i 2}-\Phi\left(\mathbf{x}_{i 2}^{\prime} \boldsymbol{\beta}\right)\right]} \\
\vdots \\
{\left[y_{i T}-\Phi\left(\mathbf{x}_{i T}^{\prime} \boldsymbol{\beta}\right)\right]}
\end{array}\right)\right]
$$

The various GMM estimators are the solutions to

$$
\boldsymbol{\beta}_{G M M, \mathbf{A}, \mathbf{W}}=\underset{\boldsymbol{\beta}}{\arg \min }\left[\mathbf{g}_{N}(\boldsymbol{\beta})\right]^{\prime} \mathbf{W}\left[\mathbf{g}_{N}(\boldsymbol{\beta})\right]
$$

\footnotetext{
${ }^{7}$ See Ahn and Schmidt (1995) and Arellano and Bover (1995) for discussion. BL constructed a different set of instruments, but did not use the strong exogeneity assumption. Their set of instruments used the current period orthogonality conditions in (7), so the total number of restrictions employed was $T K$. An expanded set of instruments based on the strict exogeneity assumption was examined in Breitung and Lechner (1997).
} 
The specific estimator is defined by the choice of instrument matrix $\mathbf{A}\left(\mathbf{X}_{i}\right)$ and weighting matrix, $\mathbf{W}$; the authors suggest several. Further details on computations appear in their paper. All of the preceding is done without (i.e., so as to avoid) direct full estimation of the matrix $\Sigma .^{8}$ Thus, the various estimators suggested attain their relative efficiencies within the class of GMM estimators, but all are inefficient relative to the full MLE implied by (4)

The authors suggest that the computation time needed to fit this model by full maximum likelihood is "prohibitively high for $T>4$ or $5 \ldots$ " For present purposes, a simple benchmark is useful. As of their writing, the mid-1990s, their computation of the "pooled" probit model using their fairly large sample of 6,350 observations and eight regressors took 30 seconds. (See their page 363.) The same computation using the same data on a less than leading edge personal computer in 2002 took less than 0.3 seconds, a more than 100 fold improvement. ${ }^{9}$ A similar comparison relates to a first step estimation of the elements of $\Sigma$, which can be done in principle by fitting a set of bivariate probit models. The authors deem this calculation "cumbersome for large $T$," but, for their sample of $N=1,270$ observations, computation of each bivariate probit model takes less than six seconds. The upshot is that computation time and "burden" for maximum likelihood and the simulation estimators, which is a recurrent theme in the BL study and one of the primary motivations for the "convenient estimators" should now be well within the acceptable range for many problems. We have fit the multivariate probit model in (3) using the full information maximum simulated likelihood estimator. The amount of computation is large, but not excessive. Estimation was fairly routine, and took altogether under an hour on a $1.66 \mathrm{Ghz}$ personal computer. Full results and details are given below.

\footnotetext{
${ }^{8}$ A considerable amount of the derivation in the paper concerns approximations to $\operatorname{Var}\left[\sqrt{N} \mathbf{g}_{N}\right]$. One additional simple estimator which was not examined would be the sample second moment matrix of the terms in $\mathbf{g}_{N}$ based on any consistent first round estimator of $\beta$, of which several were suggested. This estimator was considered in Breitung and Lechner (1997) and found to be inferior to the analytic estimator because of the very large number of overidentifying restrictions.

${ }^{9}$ The authors used Gauss ${ }^{\mathcal{O}}$ for their computations. Results in this paper were obtained with Version 8.0 of LIMDEP $P^{\odot}$.
} 


\section{Alternative forms and estimators for the panel probit model}

The panel data set used by BL contains a considerable amount of between group variation. For example, based on a simple analysis of variance, $97.6 \%$ of the variation in the FDI variable and $92.9 \%$ of that of the imports share variable are accounted for by differences in the group means. With the exception of the Butler and Moffitt random effects formulation, the approaches suggested do not model the heterogeneity that is likely to be present in a data set of this sort. Several approaches might be considered.

\subsection{Fixed and random effects estimation}

The "random effects" model analyzed by Butler and Moffitt (1982) specifies $\varepsilon_{i t}=$ $u_{i t}+v_{i}$ where $u_{i t}$ is both homoscedastic and uncorrelated across periods. A yet more restrictive approach is to assume away all the cross period correlation and treat the panel essentially as a cross section. This produces the "pooled" estimator. Under the specification in (3), both these estimators are consistent but inefficient. (Both are special cases of the GMM estimator proposed by BL.) However, the conventionally estimated standard errors that accompany each are inappropriate as they ignore the unrestricted cross period correlation.

The Butler and Moffitt random effects probit model, $\varepsilon_{i t}=u_{i t}+v_{i}$ assumes that the individual heterogeneity $v_{i}$ is uncorrelated with the included variables $\mathbf{x}_{i t}$ in all periods and is time invariant. This produces the modified covariance matrix $\sigma_{t s}=\sigma_{v}{ }^{2} /\left(\sigma_{u}{ }^{2}+\sigma_{v}{ }^{2}\right)=$ $\rho$ and $\sigma_{v}{ }^{2}+\sigma_{u}{ }^{2}=1$ on the main diagonal of $\Sigma$. In this case, the model contains only $\beta$ plus one additional correlation parameter, $\rho$. The log likelihood may be maximized by Hermite quadrature or by simulation methods. (Some details are given by BL.)

If the strict exogeneity assumption is violated, then the random effects estimator is inconsistent. In the linear regression case, an attractive alternative is the fixed effects (dummy variable) model. In the probit setting, because of the nonlinearity of the model, fixed effects estimation is more complicated than in the linear model. ${ }^{10}$ However, in applications the size of the one considered here, with $T=5$ (and $T=10$ in their Monte Carlo experiment), complexity of the computations is a moot point. Regardless of the

\footnotetext{
${ }^{10}$ Fixed effects estimation of the probit model is feasible, however, even for very large $N$. This is treated in detail in Greene (2002).
} 
true underlying formulation - even if the fixed effects model is appropriate - the maximum likelihood fixed effects estimator is inconsistent because of the incidental parameters problem. ${ }^{11}$ An additional problem with the fixed effects model is that it precludes time invariant regressors. Two of the variables in our application are time invariant. [The Hausman and Taylor (1981) approach is not useful here because the data cannot be transformed to remove the effect as in their linear regression model.] Results for a restricted fixed effects model are presented below for comparison, but it is noted that for $T=5$, the persistent bias of this estimator is substantial, and this is not suggested as a useful alternative to the random effects model. Both are problematic.

\subsection{A random parameters model}

Two alternative formulations that have been widely used to analyze panel data in other contexts, but not in the probit model, are the random parameters model and the latent class model. ${ }^{12}$

The random parameters model is an extension of (1),

$$
\begin{aligned}
& y_{i t}^{*}=\mathbf{x}_{i t}^{\prime} \beta_{i}+\varepsilon_{i t}, t=1, \ldots, T, i=1, \ldots, N, \varepsilon_{i t} \sim N[0,1] \\
& y_{i t}=\mathbf{1}\left(y_{i t}^{*}>0\right)
\end{aligned}
$$

where

$$
\begin{aligned}
& \beta_{i}=\beta+\Delta \mathbf{z}_{i}+\Gamma \mathbf{w}_{i} \\
& \beta=K \times 1 \text { vector of unconditional means } \\
& \Delta=K \times L \text { matrix unknown location parameters, } \\
& \Gamma=K \times K \text { lower triangular matrix of unknown variance parameters, } \\
& \mathbf{z}_{i}=L \times 1 \text { vector of individual characteristics } \\
& \mathbf{w}_{i}=K \times 1 \text { vector of random latent individual effects }
\end{aligned}
$$

\footnotetext{
${ }^{11}$ The encouraging results in Heckman (1981) for the fixed effects probit estimator appear to be incorrect. See Greene (2002). See Lancaster (2000) for theoretical development and Greene (2002) for empirical evidence on the incidental parameters problem in estimation of the fixed effects probit model.

${ }^{12}$ A restricted form of the random parameters probit model analyzed here was analyzed by Akin, Guilkey and Sickles (1979), Guilkey and Murphy (1993) and Sepanski (2000). The random effects model is also a special case which has been applied in a number of applications. An extensive analysis of the random parameters logit model is Train (2002). Comments also appear in McFadden and Train (2000).
} 
with

$$
\begin{aligned}
& E\left[\mathbf{w}_{i} \mid \mathbf{X}_{i}, \mathbf{Z}_{i}\right]=\mathbf{0} \\
& \operatorname{Var}\left[\mathbf{w}_{i} \mid \mathbf{X}_{i}, \mathbf{Z}_{i}\right]=\mathbf{V}=K \times K \text { diagonal matrix of known constants. }
\end{aligned}
$$

It follows that

and

$$
E\left[\beta_{i} \mid \mathbf{X}_{i}, \mathbf{z}_{i}\right]=\beta+\Delta \mathbf{z}_{i}
$$

$$
\operatorname{Var}\left[\beta_{i} \mid \mathbf{X}_{i}, \mathbf{z}_{i}\right]=\Gamma \mathbf{V} \Gamma^{\prime}=\Omega .
$$

The distribution of $\beta_{i}$ is induced by that of $\mathbf{w}_{i}$ and remains to be specified. Since $\Gamma$ need not be diagonal, in principle, the distribution could be a complicated mixture of diverse components. If $\mathbf{w}_{i}$ is assumed to have a standard, spherical normal distribution, then $\beta_{i}$ will be normally distributed with the moments given above. We use the Cholesky factorization to form the covariance matrix of the random parameters. The covariance matrix of $\mathbf{w}_{i}$ is assumed known and diagonal, since any unknown parameters will be absorbed in $\Gamma$. In most cases, when it is assumed that the random parameters are normally distributed across individuals, $\mathbf{V}$ will be an identity matrix. It is not necessary to assume normality - infinite range of variation may be implausible for certain parameters. As such, the diagonals of $\mathbf{V}$ might take other values. For example, if the variation of a parameter is assumed to follow a uniform distribution, then the corresponding value of $\mathbf{V}$ would equal 1/12 and the unknown scaling would be accounted for by the corresponding elements in $\Gamma$.

The mean vector in the random parameters model is formulated with a vector of time invariant, individual specific variables $\mathbf{z}_{i}$. When present, the unknown coefficients are contained in the corresponding row of $\Delta$. Nonrandom parameters in a model are specified by imposing the constraint that the corresponding rows of $\Gamma$ contain zeros. Allowing nonzero rows in $\Delta$ while constraining the counterpart row in $\Gamma$ to be zero provides a convenient means of formulating a 'hierarchical' model. The original random effects panel data model examined in the preceding section is produced by including in the model a simple random constant term. (The firm specific mean terms, $\Delta \mathbf{z}_{i}$ will not be present.) The dynamic effects are more difficult to accommodate. One possibility is to generate $\mathbf{w}_{i t}$ by a stochastic process, such as an AR(1). Thus, as opposed to the time 
invariant effects model, the preceding can be made dynamic by allowing $\mathbf{w}_{i t}$ to equal $\mathbf{R} \mathbf{w}_{\mathrm{i}, \mathrm{t}-1}+\mathbf{h}_{i t}$ where $\mathbf{R}$ is a diagonal matrix of autocorrelation coefficients to be estimated with the other model parameters and $\mathbf{h}_{i t}$ is the white noise process. [See Greene (2002).] Other stochastic processes could be accommodated as well.

The random parameters model may be estimated by maximum simulated likelihood or by Markov Chain Monte Carlo (MCMC) methods. ${ }^{13}$ Conditioned on $\mathbf{w}_{i}$, observations on $y_{i t}$ are independent across time - timewise correlation per the focus of this paper would arise through correlation of elements of $\beta_{i}$. The joint conditional density of the $T$ observations on $y_{i t}$ is

$$
f\left(\mathbf{y}_{i} \mid \mathbf{X}_{i}, \beta_{i}\right)=\prod_{t=1}^{T} \Phi\left[\left(2 y_{i t}-1\right) \mathbf{x}_{i t}^{\prime} \beta_{i}\right] .
$$

The contribution of this observation to the log likelihood function for the observed data is obtained by integrating the latent heterogeneity out of the distribution. Thus,

$$
\log L=\sum_{i=1}^{N} \log L_{i}=\sum_{i=1}^{N} \log \int_{\beta_{i}} \prod_{t=1}^{T} \Phi\left[\left(2 y_{i t}-1\right) \mathbf{x}_{i t}^{\prime} \beta_{i}\right] g\left(\beta_{i} \mid \beta, \Delta, \Gamma, \mathbf{z}_{i}\right) d \beta_{i}
$$

Full information maximum likelihood estimates of the parameters $\beta, \Delta, \Gamma$ are obtained by maximizing this function. Since the function involves multidimensional integration, direct optimization is generally not feasible. Maximum simulated likelihood is used instead. The simulated log likelihood is

$$
\log L_{s}=\sum_{i=1}^{N} \log L_{i s}=\sum_{i=1}^{N} \log \frac{1}{R} \sum_{r=1}^{R} \prod_{t=1}^{T} \Phi\left[\left(2 y_{i t}-1\right) \mathbf{x}_{i t}^{\prime} \beta_{i r}\right]
$$

where $\beta_{i r}$ is the $r$ th of $R$ simulated draws on $\beta_{i}=\beta+\Delta \mathbf{z}_{i}+\Gamma \mathbf{w}_{i}$ from the underlying distribution of $\mathbf{w}_{i}$. Estimates of $\beta, \Delta$, and $\Gamma$ are obtained by maximizing the simulated $\log$ likelihood function. ${ }^{14}$ Observations on $\beta_{i r}$ are constructed from primitive draws on $\mathbf{w}_{i}$. Note that it is not necessary to assume normally distributed parameters. The integration by simulation simplifies the computations in such a way that other distributions are easily

\footnotetext{
13 See, Train (2002), Greene (2003, Chapter 16), or Albert and Chib (1993).

${ }^{14}$ See Gourieroux and Monfort (1996) and Train (2002) for discussion.
} 
accommodated. For example, the range of variation can be restricted by using a "tent" or uniform distribution, either of which is easily simulated. The estimated elements of $\Gamma$ will carry the sample information on the range of variation. A more complete exposition in the context of logit models for discrete choice appears in Train (2002) and in general terms in Greene (2001). Maximum simulated likelihood estimation is extremely computation intensive. The process is speeded up by using a quasi-Monte Carlo method based on Halton sequences of draws instead of random draws using a random number generator. The method is described in the appendix.

With estimates of the structural parameters $(\beta, \Delta, \Gamma)$ in hand, estimates of the individual specific parameter vectors may be obtained by the posterior mean

$$
\hat{\boldsymbol{\beta}}_{i}=E\left[\boldsymbol{\beta}_{i} \mid \beta, \Delta, \Gamma, \mathbf{y}_{i}, \mathbf{X}_{i}, \mathbf{z}_{i}\right]=\frac{\int_{\beta_{i}} \beta_{i}\left[\prod_{t=1}^{T} \Phi\left[\left(2 y_{i t}-1\right) \mathbf{x}_{i t}^{\prime} \beta_{i}\right] g\left(\beta_{i} \mid \beta, \Delta, \Gamma, \mathbf{z}_{i}\right)\right] d \boldsymbol{\beta}_{i}}{\int_{\boldsymbol{\beta}_{i}}\left[\prod_{t=1}^{T} \Phi\left[\left(2 y_{i t}-1\right) \mathbf{x}_{i t}^{\prime} \boldsymbol{\beta}_{i}\right] g\left(\boldsymbol{\beta}_{i} \mid \beta, \Delta, \Gamma, \mathbf{z}_{i}\right)\right] d \boldsymbol{\beta}_{i}}
$$

[See Train (2002), Chapter 11.] The parts can be estimated by simulation. The application below demonstrates.

\subsection{A latent class model}

Assuming independence across time, the conditional density for the observed data is

$$
f\left(\mathbf{y}_{i} \mid \mathbf{X}_{i}, \beta_{i}\right)=\prod_{t=1}^{T} \Phi\left[\left(2 y_{i t}-1\right) \mathbf{x}_{i t}^{\prime} \beta_{i}\right]
$$

The conditional density of the random parameters, induced by the random vector $\mathbf{w}_{i}$, is $g\left(\beta_{i} \mid \beta, \Delta, \Gamma, \mathbf{z}_{i}\right)$. The unconditional density is the contribution to the likelihood given earlier,

$$
f\left(\mathbf{y}_{i} \mid \mathbf{X}_{i}, \mathbf{z}_{i}, \beta, \Delta, \Gamma\right)=E_{\beta_{i}}\left[f\left(\mathbf{y}_{i} \mid \mathbf{X}_{i}, \beta_{i}\right)\right]=\int_{\beta_{i}} \prod_{t=1}^{T} \Phi\left[\left(2 y_{i t}-1\right) \mathbf{x}_{i t}^{\prime} \beta_{i}\right] g\left(\beta_{i} \mid \beta, \Delta, \Gamma, \mathbf{z}_{i}\right) d \beta_{i} .
$$

The density of $\beta_{i}$ is the weighting distribution in this average. The preceding has assumed this is a continuous distribution. If the distribution of $\beta_{i}$ has finite, discrete support over $J$ points (classes), $p\left(\beta_{j}\right), j=1, \ldots, J$, then the resulting formulation is, 


$$
f\left(\mathbf{y}_{i} \mid \mathbf{X}_{i t}, \mathbf{z}_{i}, \Delta\right)=\sum_{j=1}^{M} p_{i j}\left(\Delta, \mathbf{z}_{i}\right) f\left(\mathbf{y}_{i} \mid \mathbf{X}_{i}, \beta_{j}\right)
$$

where it remains to parameterize the regime probabilities, $p_{i j}{ }^{15}$ (We will arrive at a discrete distribution that is parameterized in terms of only the matrix $\Delta$, so $\beta$ and $\Sigma$ will be unnecessary.) Estimation is over the $J$ regime probabilities and the regime level parameters $\beta_{j}$. The class probabilities must be constrained to sum to 1 . A simple approach is to reparameterize them as a set of logit probabilities,

$$
p_{i j}=\frac{e^{\theta_{i j}}}{\sum_{j=1}^{J} e^{\theta_{i j}}}, j=1, \ldots, J, \theta_{i J}=0, \theta_{i j}=\delta_{j}^{\prime} \mathbf{z}_{i}{ }^{16}
$$

The resulting log likelihood is a continuous function of the parameters, and maximization is straightforward.

Estimation produces values for the structural parameters, $\beta_{j}$, and the parameters of the prior class probabilities, $\delta_{j}$. One might also be interested in the posterior class probabilities,

$$
\begin{aligned}
\operatorname{Prob}(\text { class } j \mid \text { observation } i) & =\frac{f(\text { observation } i \mid \text { class } j) \operatorname{Prob}(\text { class } j)}{\sum_{j=1}^{J} f(\text { observation } i \mid \text { class } j) \operatorname{Prob}(\operatorname{class} j)} \\
& =\frac{f\left(\mathbf{y}_{i} \mid \mathbf{X}_{i}, \beta_{j}\right) p_{i j}\left(\Delta, \mathbf{z}_{i}\right)}{\sum_{j=1}^{M} f\left(\mathbf{y}_{i} \mid \mathbf{X}_{i}, \beta_{j}\right) p_{i j}\left(\Delta, \mathbf{z}_{i}\right)} \\
& =w_{i j} .
\end{aligned}
$$

This set of probabilities, $\mathbf{w}_{i}=\left(w_{i 1}, w_{i 2}, \ldots, w_{i J}\right)$ gives the posterior density over the distribution of values of $\beta$, that is, $\left[\beta_{1}, \beta_{2}, \ldots, \beta_{J}\right]$. An estimator of the individual specific parameter vector would then be the posterior mean

\footnotetext{
${ }^{15}$ Applications to models for count data may be found in Nagin and Land (1993), Wang Cockburn and Puterman (1998) and Wedel, DeSarbo, Bult, and Ramaswamy. (1993).

${ }^{16}$ As noted, this model has appeared in a number of applications to count data. Wedel et al. imposed the adding up constraint on the prior probabilities by a Lagrangean approach. As can be seen above, a simple reparameterization of the probabilities achieves the same end with much less effort. Nagin and Land (1993) and Wang et al. (1998) used the logit parameterization. Brannas and Rosenqvist (1994) forced the probabilities in their model to lie in the unit interval by using the parameterization $p_{j}=1 /\left[1+\exp \left(-\theta_{j}\right)\right]$ with $\theta_{j}$ unrestricted. This does solve the problem, but they did not impose the adding up constraint, $\Sigma_{j} p_{j}=1$ in their model; they simply estimated the first $\theta_{1}, \ldots, \theta_{M-1}$ without restriction and computed $p_{M}$ residually, a procedure that is not guaranteed to succeed.
} 


$$
\hat{\boldsymbol{\beta}}_{i}^{p}=\hat{E}_{j}\left[\boldsymbol{\beta}_{j} \mid \text { observation } i\right]=\sum_{j=1}^{J} w_{i j} \hat{\boldsymbol{\beta}}_{j}
$$

The EM algorithm is a convenient approach for estimation of this model. ${ }^{17}$ Let $d_{i j}$ $=1$ if individual $i$ is a member of class $j$ and zero otherwise. We treat $d_{i j}$ as missing data to be estimated. The joint density of $J d_{i j}$ is multinomial with probabilities $p_{i j}$;

$$
f\left(d_{i 1}, d_{i 2}, \ldots, d_{i J} \mid \mathbf{z}_{i}, \Delta\right)=f\left(\mathbf{d}_{i} \mid \mathbf{z}_{i}, \Delta\right)=\prod_{j=1}^{J} p_{i j}^{d_{i j}} .
$$

The complete data log likelihood is built from the joint density,

$$
f\left(\mathbf{y}_{i}, \mathbf{d}_{i} \mid \mathbf{X}_{i}, \mathbf{z}_{i}, \beta, \Delta\right)=f\left(\mathbf{y}_{i} \mid \mathbf{d}_{i}, \mathbf{X}_{i}, \beta\right) f\left(\mathbf{d}_{i} \mid \mathbf{Z}_{i}, \Delta\right) .
$$

Thus,

$$
\log L_{c}=\sum_{i=1}^{N} \sum_{j=1}^{J}\left[d_{i j} \log f\left(\mathbf{y}_{i} \mid \mathbf{X}_{i}, \boldsymbol{\beta}_{j}\right)+d_{i j} \log p_{i j}\right]
$$

The expectation $(E)$ step of the process involves obtaining the expectation of this log likelihood conditioned over the unobserved data. This involves replacing the $d_{i j} \mathrm{~s}$ in log $L_{c}$ with the posterior probabilities, $w_{i j}$, derived above (computed at the current estimates of the other parameters). The maximization $(M)$ step then involves maximizing the resulting conditional log likelihood with these estimated posterior probabilities treated as known. Conditioned on the posteriors, $E\left[\log L_{c}\right]$ factors into two parts that may be maximized separately. By construction, $\Sigma_{j} w_{i j}=1$. The first part of the log likelihood becomes a weighted log likelihood with known weights for which the likelihood equations are

$$
\frac{\partial E\left[\log L_{c}\right]}{\partial \boldsymbol{\beta}_{j}}=\sum_{i=1}^{N} w_{i j} \frac{\partial \log f\left(\mathbf{y}_{i} \mid \mathbf{X}_{i}, \boldsymbol{\beta}_{j}\right)}{\partial \boldsymbol{\beta}_{j}}=\mathbf{0} .
$$

This involves simply maximizing a weighted, pooled log likelihood for each class parameter vector. If there are no individual specific variables in the probabilities, then the maximum likelihood estimators of the class probabilities are just the sample averages of the estimated weights;

\footnotetext{
${ }^{17}$ See Dempster, Laird, and Rubin (1977) and Wedel et al. (1993).
} 


$$
\hat{p}_{j}=\frac{\sum_{i=1}^{N} w_{i j}}{N}
$$

If the logistic parameterization has been used, then the conditional log likelihood function at the $M$ step for these parameters is a weighted multinomial logit log likelihood, which will require an iterative solution. The iterative solution for the structural parameters, $\delta_{j}$ is the solutions to the likelihood equations

$$
\frac{\partial E\left[\log L_{c}\right]}{\partial \boldsymbol{\delta}_{j}}=\sum_{i=1}^{N}\left(w_{i j}-\hat{p}_{i j}\right) \mathbf{z}_{i}=\mathbf{0} .
$$

This is the first order conditions for the multinomial logit model with proportion rather than individual data for the dependent variable (the weights). The EM method thus amounts to cycling between these two steps - computing the parameters for the class probabilities via the multinomial logit procedure at the second step above, then recomputing the class specific weighted probit estimators at the first step by a simple weighted, pooled maximum likelihood probit estimator.

\section{Application}

Bertschek and Lechner applied the GMM estimator to an analysis of the product innovation activity of 1,270 German firms observed in five years, 1984 - 1988, in response to imports and foreign direct investment. [See Bertschek (1995).] The basic model to be estimated is a probit model based on the latent regression

$$
y_{i t}^{*}=\beta_{1}+\sum_{k=2}^{8} x_{k, i t} \beta_{k}+\varepsilon_{i t}, y_{i t}=\mathbf{1}\left(y_{i t}^{*}>0\right), i=1, \ldots, 1270, t=1984, \ldots, 1988 .
$$

where

$y_{i t}=1$ if a product innovation was realized by firm $\mathrm{i}$ in year $\mathrm{t}, 0$ otherwise,

$x_{2, i t}=$ Log of industry sales in DM,

$x_{3, i t}=$ Import share $=$ ratio of industry imports to (industry sales plus imports),

$x_{4, i t}=$ Relative firm size $=$ ratio of employment in business unit to employment in the industry (times 30),

$x_{5, i t}=$ FDI share $=$ Ratio of industry foreign direct investment to (industry sales, plus imports),

$x_{6, i t}=$ Productivity $=$ Ratio of industry value added to industry employment,

$x_{7, i t}=$ Raw materials sector $=1$ if the firm is in this sector,

$x_{8, i t}=$ Investment goods sector $=1$ if the firm is in this sector, 
Descriptive statistics and further discussion appear in the earlier papers. Their primary interest was in the effect of imports and inward foreign direct investment on innovation. Both are hypothesized to have a positive effect. We have extended the analysis with their data.

Table 1 presents the base case, "pooled" estimator. This is the simple probit estimator that treats the entire sample as if it were a large cross section. This estimator is consistent, but inefficient. Four sets of asymptotic standard errors are presented with the estimates. The first are BL's reported standard errors. These are computed using a robust estimator based on White (1982). [See their pp. 345 and 359.) The second set are the square roots of the diagonals of the negative of the inverse of the Hessian computed at the maximum likelihood estimates (which we have reproduced). The third set are those computed by BL based on Avery, Hansen and Hotz's (1983) GMM estimator. A natural, simpler alternative which should be appropriate in this setting is the so-called "cluster" estimator [See Stata (1998)]:

$$
\begin{aligned}
& \hat{\mathbf{V}}(\hat{\boldsymbol{\beta}})=\left(\frac{N}{N-1}\right)\left(-\mathbf{H}^{-1}\right)\left(\sum_{i=1}^{n} \mathbf{g}_{i} \mathbf{g}_{i}^{\prime}\right)\left(-\mathbf{H}^{-1}\right) \\
& \mathbf{g}_{i}=\sum_{t=1}^{T} \mathbf{g}_{i t}
\end{aligned}
$$

The matrix $\mathbf{H}$ is the conventional Hessian of the maximized $\log$ likelihood and $\mathbf{g}_{i t}$ is the derivative of the individual term in the pooled log likelihood. As can be seen in Table 1, the cluster estimator produces essentially the same results as the GMM based estimator. The last two columns of Table 1 present the estimated partial effects of the variables on the probability of a realized innovation. Standard errors are based on the cluster estimator and are computed using the delta method. [See Greene (2003, chapter 19).] The estimates for the sector dummy variables are computed by evaluating the probability at the means of the other variables and with the dummy variables equal to one then zero the marginal effect in each case is the difference. The results thus far are consistent with the hypothesis that imports and FDI positively and significantly affect product innovation. 
Table 1. Estimated Pooled Probit Model

\begin{tabular}{|c|c|c|c|c|c|c|c|c|}
\hline & & \multicolumn{4}{|c|}{ Estimated Standard Errors } & \multicolumn{3}{|c|}{ Marginal Effects } \\
\hline Variable & Estimate $^{\mathrm{a}}$ & $\operatorname{se}(1)^{b}$ & $\operatorname{se}(2)^{c}$ & $\operatorname{se}(3)^{\mathrm{d}}$ & $\operatorname{se}(4)^{\mathrm{e}}$ & Partial $^{\mathrm{f}}$ & Std. Err. & $t$ ratio \\
\hline Constant & -1.960 & 0.21 & 0.230 & 0.377 & 0.373 & - & - & - \\
\hline $\log$ Sales & 0.177 & 0.025 & 0.0222 & 0.0375 & 0.0358 & 0.0683 & 0.0138 & 4.96 \\
\hline Rel Size & 1.072 & 0.21 & 0.142 & 0.306 & 0.269 & 0.413 & 0.103 & 4.01 \\
\hline Imports & 1.134 & 0.15 & 0.151 & 0.246 & 0.243 & 0.437 & 0.0938 & 4.66 \\
\hline FDI & 2.853 & 0.47 & 0.402 & 0.679 & 0.642 & 1.099 & 0.247 & 4.44 \\
\hline Prod. & -2.341 & 1.10 & 0.715 & 1.300 & 1.115 & -0.902 & 0.429 & -2.10 \\
\hline Raw Mtl & -0.279 & 0.097 & 0.0807 & 0.133 & 0.126 & $-0.110^{\mathrm{g}}$ & 0.0503 & -2.18 \\
\hline Inv Good & 0.188 & 0.040 & 0.0392 & 0.0630 & 0.0628 & $0.0723^{\mathrm{g}}$ & 0.0241 & 3.00 \\
\hline
\end{tabular}

${ }^{\mathrm{a}}$ Recomputed. Only two digits were reported in the earlier paper.

${ }^{\mathrm{b}}$ Obtained from results in Bertschek and Lechner, Table 10.

${ }^{c}$ Square roots of the diagonals of the negative inverse of the Hessian

d Based on the Avery et al. GMM estimator

${ }^{\mathrm{e}}$ Based on the cluster estimator.

${ }^{\mathrm{f}}$ Coefficient scaled by the density evaluated at the sample means

${ }^{\mathrm{g}}$ Computed as the difference in the fitted probability with the dummy variable equal to one then zero.

Table 2 reports the estimates of the random and fixed effects models. The random effects model was fit by two methods, first using Butler and Moffitt's method with a 32 point Hermite quadrature, then by specifying the random parameters model with only a random constant term. In terms of the estimates of the parameters, it is clear that, as expected, the two integration methods produce essentially the same results. The random parameter estimator produced an estimate of the standard deviation for the random constant of 1.1707. Based on the normalization $\sigma_{v}{ }^{2}+\sigma_{u}{ }^{2}=1$, this produces an estimator of the correlation coefficient of $1.1707^{2} /\left(1+1.1707^{2}\right)=0.578$, which is identical to the estimate produced by the quadrature method. The value itself is also striking, as will be evident in the next set of results for the multivariate probit model. The fixed effects estimates in the last column display the expected large biases. Given that $T$ is only 5 in this application, the apparent distortion in the fixed effects estimator is to be expected. 
Table 2. Estimated Common Effects Models

\begin{tabular}{|l|c|c|c|c|c|c|}
\hline & \multicolumn{4}{|c|}{ Random Effects } & \multicolumn{2}{c|}{ Fixed Effects } \\
\hline & \multicolumn{2}{|c|}{ Quadrature Estimator } & \multicolumn{2}{|c|}{ Simulation Estimator } & \multicolumn{2}{c|}{} \\
\hline Variable & Estimate & Std.Error & Estimate & Std.Error & Estimate & Std. Error \\
\hline Constant & -2.839 & 0.533 & -2.884 & 0.543 & - & - \\
\hline log Sales & 0.244 & 0.0522 & 0.249 & 0.0510 & -0.6497 & 0.356 \\
\hline Rel Size & 1.522 & 0.257 & 1.452 & 0.281 & 0.278 & 0.734 \\
\hline Imports & 1.779 & 0.360 & 1.796 & 0.360 & 3.503 & 2.924 \\
\hline FDI & 3.652 & 0.870 & 3.724 & 0.831 & -8.131 & 3.381 \\
\hline Prod. & -2.307 & 1.911 & -2.321 & 0.151 & 5.300 & 4.034 \\
\hline Raw Mtl & -0.477 & 0.202 & -0.469 & 0.186 & - & - \\
\hline Inv Good & 0.331 & 0.0952 & 0.331 & 0.0915 & - & - \\
\hline$\rho$ & 0.578 & 0.0189 & $0.578^{\mathrm{a}}$ & 0.0231 & - & - \\
\hline
\end{tabular}

${ }^{\mathrm{a}}$ Based on estimated standard deviation of the random constant of 1.1707 with estimated standard error of 0.01865 .

Table 3 reports the full maximum likelihood estimates of the 5-variate probit model with homoscedasticity and the same coefficient vector in every year. This model was estimated using the GHK simulator and 50 replications. ${ }^{18}$ Derivatives of the $\log$ likelihood were computed numerically, and the BHHH (outer product of gradients) estimator was used to compute the standard errors. The estimates are very similar to the GMM estimator. The estimated standard errors are slightly smaller in most cases, as might be expected. In theory, this is the fully efficient estimator, so the discrepancies from this relationship are finite sample variation. A striking aspect of these results is the uniformity of the correlation coefficients. It appears that the random effects model is a reasonable specification for these data. The log likelihood functions are -3535.55 for the random effects model and -3522.85 for the unrestricted model. Based on these values, the chi-squared statistic for testing the nine restrictions of the equicorrelated case would be 25.4. The critical value from the chi-squared table is 16.9 for $95 \%$ significance and 21.7 for $99 \%$, so the equicorrelated case would be rejected. However, the statistic is not overwhelming. Moreover, the log likelihood for the FIML case is simulated, so it must be viewed as only approximate. The upshot is that while the simple random effects

\footnotetext{
${ }^{18}$ Estimation took roughly an hour, and convergence to this set of results was smooth and routine in 27 Broyden/Fletcher/Goldfarb/Shanno iterations. Starting values for the values reported were the pooled probit estimators for the slopes and zeros for all correlation coefficients. The estimator was restarted at the random effects estimators - all correlation coefficients equal to 0.578 and the quadrature estimated random effects slopes. The estimates thus obtained differed only marginally from those reported. Random numbers for the GHK simulator were produced using L'Ecuyer's (1998) MRG32K3A multiple recursive generator. This generator has been shown to have excellent properties and has a period of about $2^{191}$ draws before recycling.
} 
model is rejected, the statistical difference from the restrictive random effects model is less than compelling.

Table 3. Estimated Multivariate Probit Model

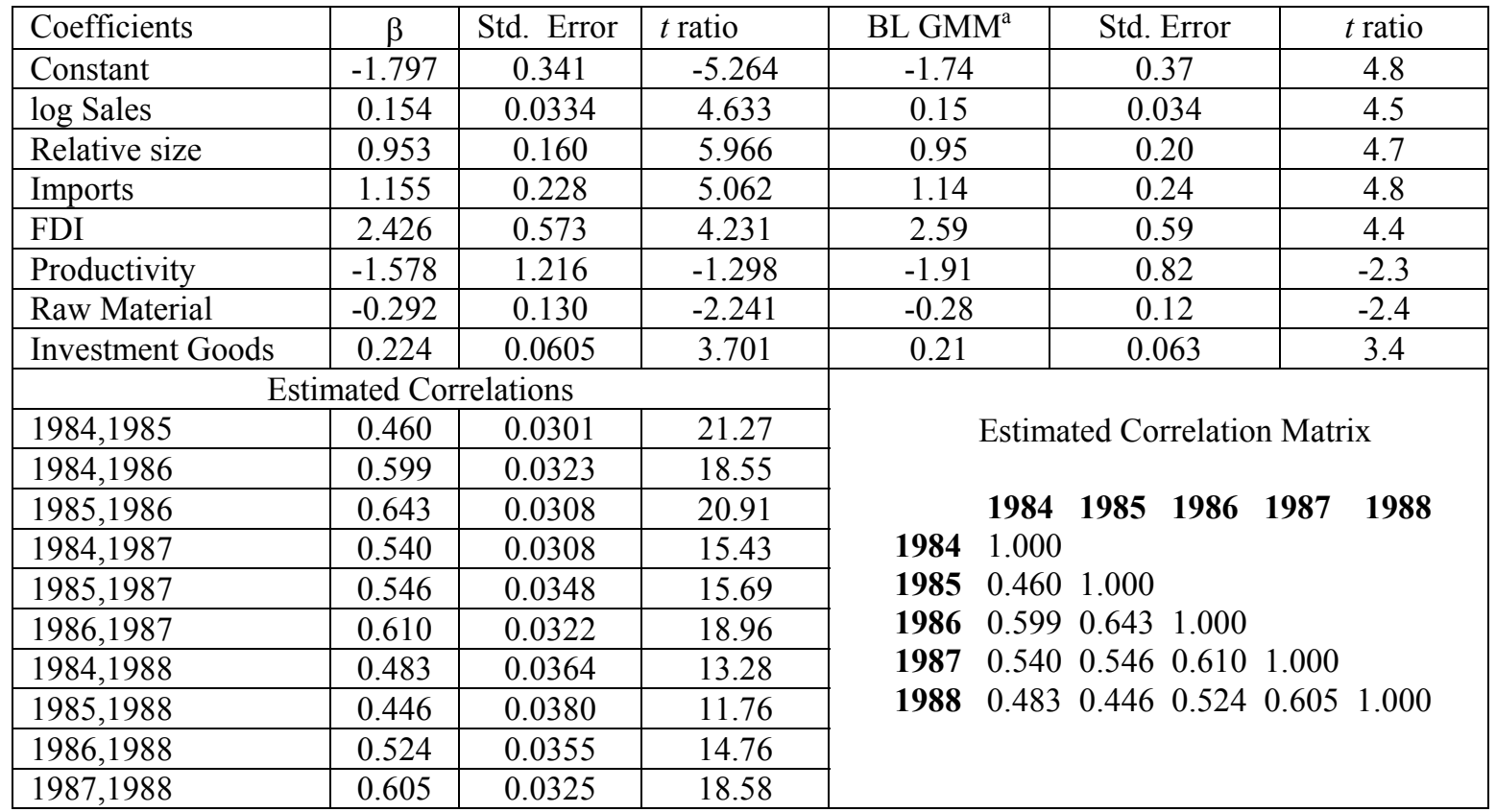

${ }^{a}$ Estimates are BL's WNP-joint uniform estimates with $k=880$. Estimates and $\mathrm{t}$ ratios from their Table 9, standard errors from their Table 10.

To explore a variant of the model based on the hypothesis of interest in the earlier paper, we fit a random parameters model of the following form:

$$
\begin{aligned}
& y_{i t}^{*}=\beta_{1 i}+\beta_{2} \text { logSales }+\beta_{3} \text { RelSize }+\beta_{4} \text { Prod }+\beta_{5 i} \text { Imports }+\beta_{6 i} \text { FDI }+\varepsilon_{i t} \\
& \left(\begin{array}{l}
\beta_{1 i} \\
\beta_{5 i} \\
\beta_{6 i}
\end{array}\right)=\left(\begin{array}{l}
\beta_{1} \\
\beta_{5} \\
\beta_{6}
\end{array}\right)+\left[\begin{array}{ll}
\delta_{1 R} & \delta_{1 I} \\
\delta_{5 R} & \delta_{1 R} \\
\delta_{1 R} & \delta_{1 R}
\end{array}\right]\left(\begin{array}{l}
\text { RawMtl } \\
\text { InvGood }_{i}
\end{array}\right)+\left[\begin{array}{ccc}
\gamma_{11} & 0 & 0 \\
\gamma_{11} & \gamma_{11} & 0 \\
\gamma_{11} & \gamma_{11} & \gamma_{11}
\end{array}\right]\left(\begin{array}{c}
w_{1 i} \\
w_{5 i} \\
w_{6 i}
\end{array}\right) \\
& \left(\begin{array}{l}
\varepsilon_{i t} \\
w_{1 i} \\
w_{5 i} \\
w_{6 i}
\end{array}\right) \sim N\left[\mathbf{0}, \mathbf{I}_{4}\right] \\
& y_{i t}=1\left(y_{i t}^{*}>0\right) .
\end{aligned}
$$


Table 4 reports estimates of the parameters of this model. The simulated log likelihood was constructed using 100 Halton draws for the replication (see the appendix). The structural parameter estimates are given in the table. The implied estimates of the variances of the parameter distributions are the square roots of the sums of squares of the elements in the corresponding rows of $\Gamma$. The implied standard deviations of the two parameter distributions might appear large in comparison to the estimated standard errors from the preceding models, but these are not the variances of the sampling distributions of the estimators. They are estimates of the variances of the distributions of these parameters across the firms in the sample. To examine this further, Figures 1 and 2 are kernel density plots of the posterior estimates of the firm specific estimates of $\beta_{5 i}$ and $\beta_{6 i}$. The graphical and statistical evidence continues to support the hypothesis that imports and FDI exert a generally positive and large influence on the probability of product innovation for these firms. Descriptive statistics for the individual specific estimates of the two parameters are given in Table 5.

Table 4. Estimated Random Parameters Model

\begin{tabular}{|l|c|c|}
\hline Variable & Estimate & Standard Error \\
\hline log Sales & 0.183 & 0.0176 \\
\hline Relative Size & 1.669 & 0.0992 \\
\hline Productivity & -3.564 & 0.520 \\
\hline \multicolumn{2}{|c|}{ Random Parameter: Constant } \\
\hline$\beta_{1}$ & -2.006 & 0.192 \\
\hline$\delta_{1 \mathrm{R}}$ & -1.259 & 0.211 \\
\hline$\delta_{1 \mathrm{I}}$ & -0.0302 & 0.0807 \\
\hline$\gamma_{11}$ & 1.439 & 0.0436 \\
\hline Implied standard deviation & 1.439 & 0.0346 \\
\hline \multicolumn{2}{|c|}{ Random Parameter: Imports } \\
\hline$\beta_{5}$ & 1.486 & 0.143 \\
\hline$\delta_{5 \mathrm{R}}$ & -0.248 & 0.394 \\
\hline$\delta_{5 \mathrm{I}}$ & 1.703 & 0.504 \\
\hline$\gamma_{51}$ & 1.402 & 0.137 \\
\hline$\gamma_{55}$ & 2.140 & 0.0735 \\
\hline Implied standard deviation & 2.558 & 0.0881 \\
\hline \multicolumn{1}{|c|}{ Random Parameter: FDI } \\
\hline$\beta_{6}$ & 2.511 & 0.309 \\
\hline$\delta_{6 \mathrm{R}}$ & 19.20 & 2.02 \\
\hline$\delta_{6 \mathrm{I}}$ & 0.648 & 1.881 \\
\hline$\gamma_{61}$ & 1.013 & 0.317 \\
\hline$\gamma_{65}$ & -3.496 & 0.286 \\
\hline$\gamma_{66}$ & 2.592 & 0.218 \\
\hline Implied Standard deviation & 4.468 & 0.268 \\
\hline
\end{tabular}


Table 5. Sample Descriptive Statistics for Individual Specific Parameters

\begin{tabular}{|l|c|c|c|c|}
\hline Variable & Mean & Std. Deviation & Minimum & Maximum \\
\hline Imports & 2.345 & 1.172 & -1.254 & 5.326 \\
\hline FDI & 4.467 & 5.557 & -2.724 & 26.916 \\
\hline
\end{tabular}

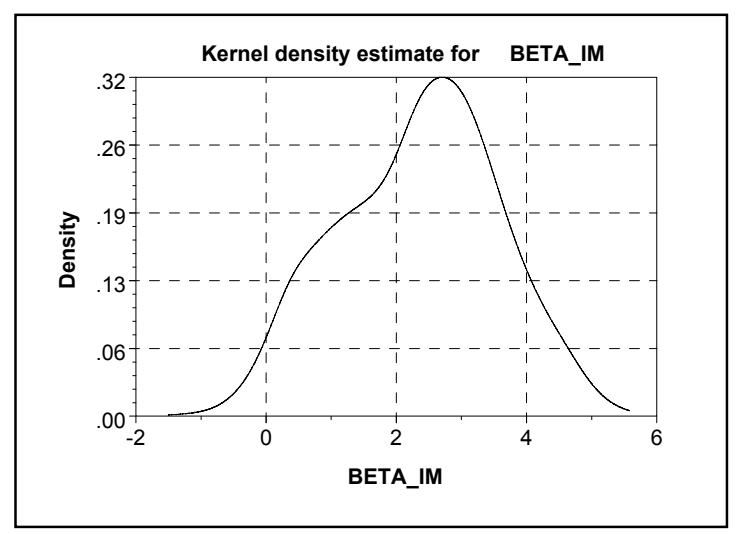

Figure 1. Kernel density plot for $\beta_{5 \mathrm{i}}$ based on the random parameters model. The bandwidth is 0.2526 using a logistic kernel.

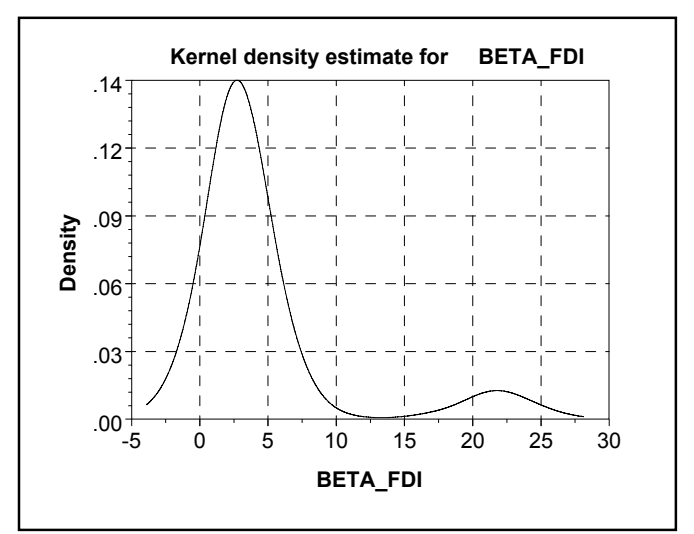

Figure 2. Kernel Density Plot for $\beta_{6 i}$ based on the random parameters model. The bandwidth is 1.1976 using a logistic kernel.

Table 6 presents the latent class estimates of the full model. Working down from $J=5$, we found that the estimates stabilized at $J=3$. There is a large amount of variation across the three classes. Little can be inferred from the particular estimates from any one class. The sample means of the posterior estimates are more revealing. As in all other cases, the original conclusion, that FDI and imports positively affect the probability of product innovation continues to be supported. The kernel density plots in Figures 3 and 4 , however, suggest a large range of variation of these effects.

Table 6. Estimated Latent Class Model

\begin{tabular}{|c|c|c|c|c|c|c|c|c|c|c|}
\hline & \multicolumn{6}{|c|}{ Class Specific Parameter Estimates } & & & & \\
\hline & \multicolumn{2}{|c|}{ Class 1} & \multicolumn{2}{|c|}{ Class 2} & \multicolumn{2}{|c|}{ Class 3} & \multicolumn{4}{|c|}{ Sample Posterior Estimates } \\
\hline & Est. & S.E. & Est. & S.E. & Est. & S.E. & Mean & S.D. & Min & Max \\
\hline Constant & -2.21 & 0.516 & -13.6 & 4.16 & -2.02 & 0.759 & -4.16 & 3.50 & -13.51 & -2.03 \\
\hline logSales & 0.308 & 0.0490 & 0.897 & 0.302 & 0.186 & 0.0714 & 0.374 & 0.187 & 0.186 & 0.889 \\
\hline RelSize & 4.21 & 0.840 & 0.904 & 0.699 & 0.529 & 0.416 & 2.53 & 1.45 & 0.529 & 4.21 \\
\hline Imports & 1.05 & 0.354 & 6.480 & 2.44 & 2.16 & 0.483 & 2.33 & 1.67 & 1.05 & 6.43 \\
\hline FDI & 2.56 & 1.172 & 11.5 & 3.10 & 2.17 & 1.07 & 4.01 & 2.76 & 2.17 & 11.43 \\
\hline Prod & -5.80 & 1.933 & 0.960 & 1.84 & -9.00 & 2.90 & -5.57 & 2.34 & -8.99 & 0.850 \\
\hline \multicolumn{7}{|c|}{ Estimated Class Probabilities Model ${ }^{\mathrm{a}}$} & & & & \\
\hline$\theta_{1}$ & 0.319 & 0.192 & -0.514 & 0.211 & $0.000^{\mathrm{b}}$ & 0.000 & & & & \\
\hline$\theta_{\mathrm{R}}$ & 0.0392 & 0.517 & 0.768 & 0.635 & 0.000 & 0.000 & & & & \\
\hline$\theta_{\mathrm{I}}$ & 0.458 & 0.221 & -0.283 & 0.303 & 0.000 & 0.000 & & & & \\
\hline Avg P & \multicolumn{2}{|c|}{0.528} & \multicolumn{2}{|c|}{0.168} & \multicolumn{2}{|c|}{0.304} & & & & \\
\hline
\end{tabular}

${ }^{a}$ Estimates are parameters of the multinomial logit model

${ }^{\mathrm{b}}$ Constrained to this value 


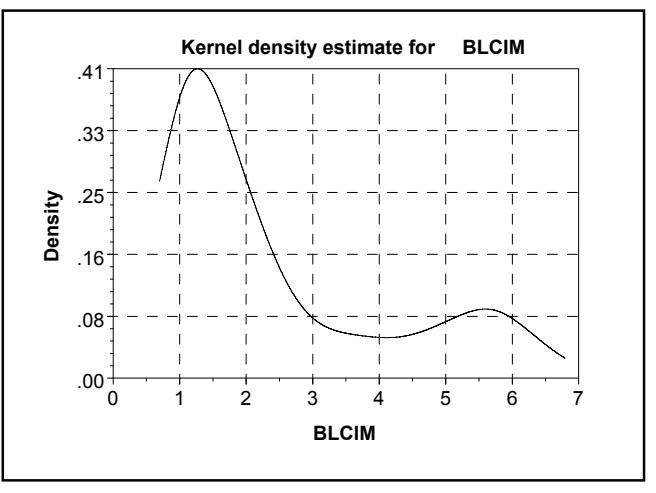

Figure 3. Kernel density plot for $\beta_{5 \mathrm{i}}$ based on the latent class model. The bandwidth is 0.3616 using a logistic kernel.

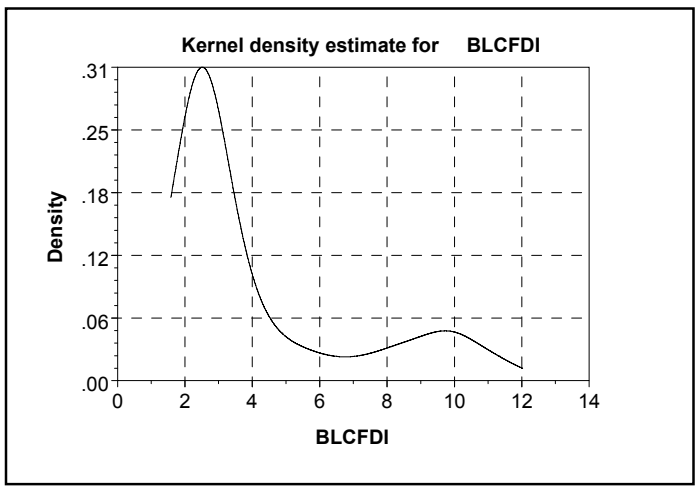

Figure 4. Kernel density plot for $\beta_{6 i}$ based on the latent class model. The bandwidth is 0.5952 using a logistic kernel.

\section{Conclusions}

The preceding has examined a number of estimators for the panel probit model. The GMM estimator appears to perform fairly well compared to the maximum likelihood estimator. On the other hand, the difficulty of obtaining the latter is considerably smaller than the discussion in BL would suggest. Part of this stems from the much greater speeds attainable with the current vintage of computers. However, it also appears that the authors were unduly pessimistic about the feasibility of using simulation methods for computation of the multivariate normal integrals needed for FIML estimation. Obviously, it is unclear what sample sizes should be used as the benchmarks for this discussion. However, their application, with $N=1,270, K=8$ and $T=5$, is reasonably large enough that one should be able to obtain some guidance from our results.

We also presented two alternative models for the panel based discrete choice analysis. These are not simple alternatives to the panel probit model suggested by BL. The random parameters model and latent class model are more elaborate model specifications that allow the analyst to glean from a rich panel such as this one information about individual heterogeneity that is neglected by the simple model formulation suggested by BL. The computation of the latent class model is exceedingly simple. Computation of the model reported here took less than 20 seconds. The random parameters model is much more computationally intensive as it requires maximization of 
a simulated log likelihood function. We find that use of a quasi Monte Carlo method, specifically Halton sequences as an alternative to simulated random sampling, greatly speeds up the process.

\section{Appendix. Quasi Monte Carlo Integration}

Gourieroux and Monfort (1996) provide the essential statistical background for the simulated maximum likelihood estimator. We assume that the original maximum likelihood estimator as posed with the intractable integral is otherwise regular - if computable, the MLE would have the familiar properties, consistency, asymptotic normality, asymptotic efficiency, and invariance to smooth transformation. Let $\theta$ denote the full vector of unknown parameters being estimated and let $\mathbf{q}_{M L}$ denote the maximum likelihood estimator of the full parameter vector shown above, and let $\mathbf{q}_{S M L}$ denote the simulated maximum likelihood (SML) estimator. Gourieroux and Monfort establish that if $\sqrt{N} / R \rightarrow 0$ and $R$ and $N \rightarrow \infty$, then $\sqrt{N}\left(\mathbf{q}_{S M L}-\theta\right)$ has the same limiting normal distribution with zero mean as $\sqrt{N}\left(\mathbf{q}_{M L}-\theta\right)$. That is, under the assumptions, the maximum simulated likelihood estimator and the true maximum likelihood estimator are asymptotically equivalent. The requirement that the number of draws, $R$, increase faster than the square root of the number of observations, $N$, is important to their result. The requirement is easily met by tying $R$ to the sample size, as in, for example, $R=N^{.5+\delta}$ for some positive $\delta$. There does remain a finite $R$ bias in the estimator, which the authors obtain as approximately equal to $(1 / R)$ times a vector which is a finite vector of constants (see p. 44). Generalities are difficult, but the authors suggest that when the MLE is relatively "precise," the bias is likely to be small. In Munkin and Trivedi's (2000) Monte Carlo study of the effect, in samples of 1000 and numbers of replications around 200 or 300 , the bias of the simulation based estimator appears to be trivial.

The results thus far are based on random sampling from the underlying distribution of $\mathbf{w}$. But, the simulation method, itself, contributes to the variation of SML estimator. [See, e.g., Geweke (1995).] Judicious choice of the random draws for the simulation can be helpful in speeding up the convergence. [See Bhat (1999).] One technique commonly used is antithetic sampling. [See Geweke $(1995,1998)$ and Ripley (1987).] The technique involves sampling $R / 2$ independent pairs of draws where the 
members of the pair are negatively correlated. One technique often used, for example is to pair each draw $\mathbf{w}_{i r}$ with $-\mathbf{w}_{i r}$. (A loose end in the discussion at this point concerns what becomes of the finite simulation bias in the estimator. The results in Gourieroux and Monfort hinge on random sampling.)

Quasi Monte Carlo (QMC) methods are based on replacing the pseudo random draws of the Monte Carlo integration with a grid of "cleverly" selected points which are nonrandom but provide more uniform coverage of the domain of the integral. The logic of the technique is that randomness of the draws used in the integral is not the objective in the calculation. Convergence of the average to the expectation (integral) is, and this can be achieved by other types of sequences. A number of such strategies is surveyed in Bhat (1999), Sloan and Wozniakowski (1998) and Morokoff and Caflisch (1995). The advantage of QMC as opposed to MC integration is that for some types of sequences, the accuracy is far greater, convergence is much faster and the simulation variance is smaller. For the one we will advocate here, Halton sequences, Bhat (1995) found relative efficiencies of the QMC method to the MC method in moderately sized estimation problems to be on the order of ten or twenty to one.

Monte Carlo simulation based estimation uses a random number generator to produce the draws from a specified distribution. The central component of the approach is draws from the standard continuous uniform distribution, $U[0,1]$. Draws from other distributions are obtained from these by using the inverse probability transformation. In particular, if $u_{i}$ is one draw from $U[0,1]$, then $v_{i}=\Phi^{-1}\left(u_{i}\right)$ produces a draw from the standard normal distribution; $v_{i}$ can then be unstandardized by the further transformation $z_{i}=\sigma v_{i}+\mu$. Draws from other distributions used, e.g., in Train (1999) are the uniform $[-1,1]$ distribution for which $v_{i}=2 u_{i}-1$ and the tent distribution, for which $v_{i}=\sqrt{2 u_{i}}-1$ if $u_{i} \leq 0.5, v_{i}=1-\sqrt{2 u_{i}-1}$ otherwise. Geweke (1995), and Geweke, Hajivassiliou, and Keane (1994) discuss simulation from the multivariate truncated normal distribution with this method.

The Halton sequence is generated as follows: Let $r$ be a prime number larger than 2. Expand the sequence of integers $g=1, \ldots$ in terms of the base $r$ as 


$$
g=\sum_{i=0}^{I} b_{i} r^{i} \text { where by construction, } 0 \leq b_{i} \leq r-1 \text { and } r^{I} \leq g<r^{I+1} \text {. }
$$

The Halton sequence of values that corresponds to this series is

$$
H_{r}(g)=\sum_{i=0}^{I} b_{i} r^{-i-1}
$$

For example, using base 5 , the integer 37 has $b_{0}=2, b_{1}=2$, and $b_{3}=1$. Then

$$
H_{5}(37)=2 \times 5^{-1}+2 \times 5^{-2}+1 \times 5^{-3}=0.448 .
$$

The sequence of Halton values is efficiently spread over the unit interval. The sequence is not random as the sequence of pseudo-random numbers is. Figures 5 and 6 below show two sequences of 1,000 Halton draws based on $r=7$ and $r=9$ and two sequences of 1,000 psuedorandom draws. The clumping evident in the figure on the left is the feature that necessitates large pseudo-samples for simulations.

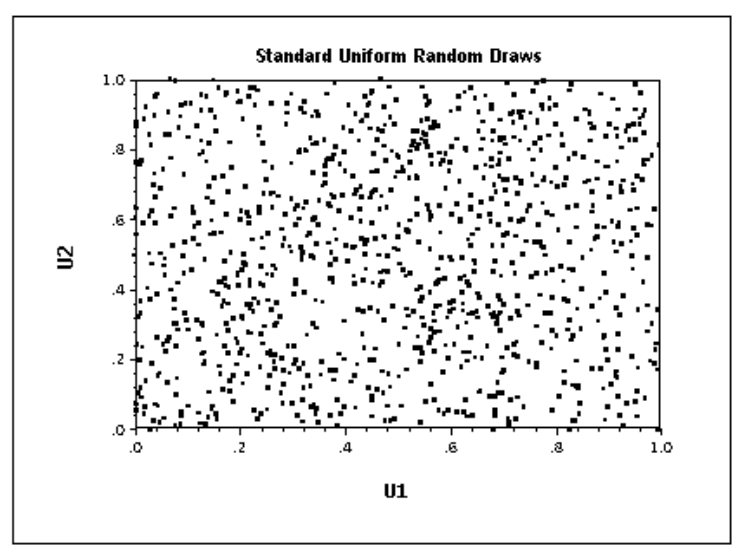

Figure 5. Random draws from $\mathrm{U}(0,1)$

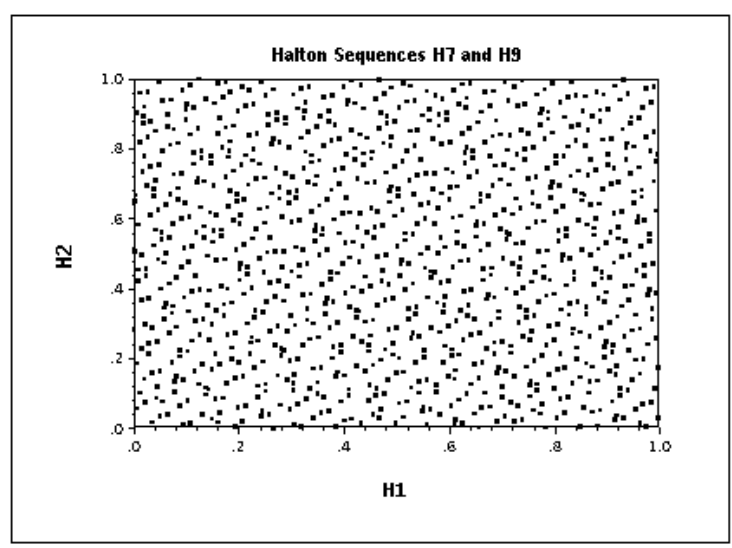

Figure 6. Two Halton sequences 


\section{References}

Ahn, S. and P. Schmidt, "Efficient Estimation of Models for Dynamic Panel Data," Journal of Econometrics, 68, 1995, pp. 3-38.

Akin, J., D. Guilkey and R. Sickles, A Random Coefficient Probit Model with an Application to a Study of Migration," Journal of Econometrics, 11, 1979, pp. 233-246.

Albert, J. and S. Chib, "Bayesian Analysis of Binary and Polytomous data," Journal of The American Statistical Association, 88, 1993, pp. 669-679.

Arellano, M. and O. Bover, "Another Look at the Instrumental Variable Estimation of Error-Components Models," Journal of Econometrics, 68, 1995, pp. 29-51.

Avery, R., L. Hansen and J. Hotz, "Multiperiod Probit Models and Orthogonality Condition Estimation," International Economic Review, 24, 1983, pp. 21-35.

Bertschek, I., "Product and Process Innovation as a Response to increasing Imports and Foreign Direct Investment, Journal of Industrial Economics, 43, 4, 1995, pp. 341-357.

Bertschek, I. and M. Lechner, "Convenient Estimators for the Panel Probit Model," Journal of Econometrics, 87,2, 1998, pp. 329-372.

Bhat, C., "Quasi-Random Maximum Simulated Likelihood Estimation of the Mixed Multinomial Logit Model," Manuscript, Department of Civil Engineering, University of Texas, Austin, 1999.

Boyes, W., Hoffman, D. and S. Low, "An Econometric Analysis of the Bank Credit Scoring Problem," Journal of Econometrics, 40, 1989, pp. 3-14.

Brannas, K. and G. Rosenqvist, "Semiparametric Estimation of Heterogeneous Count Data Models," European Journal of Operational Research, 76, 1994, pp. 247-258.

Breitung, J. and M. Lechner, "GMM Estimation of Nonlinear Models on Panel Data," Humboldt University, SFB, Discussion paper, 67, 1995; "Estimation de modeles non lineaires, sur donnees de panel par la methode des moments generalises," Economie et Prevision, 1997.

Burnett, N., "Gender Economics Courses in Liberal Arts Colleges," Journal of Economic Education, 24, 8, 1997, pp. 369-377.

Butler, J. and R. Moffitt, "A Computationally Efficient Quadrature Procedure for the One Factor Multinomial Probit Model," Econometrica, 50, 1982, pp. 761-764.

Dempster, A., N. Laird and D. Rubin, "Maximum Likelihood From Incomplete Data via the E.M. Algorithm," Journal of the Royal Statistical Society, Series B, 39, 1, 1977, pp. 1-38.

Geweke, J., "Antithetic Acceleration of Monte Carlo Integration in Bayesian Inference," Journal of Econometrics, 38, 1988, pp. 73-89.

Geweke, J., "Monte Carlo Simulation and Numerical Integration," Staff Research Report 192, Federal Reserve Bank of Minneapolis, 1995.

Geweke, J., "Posterior Simulators in Econometrics," in Kreps, D. and K. Wallis, eds., Advances in Statistics and Econometrics: Theory and Applications, Vol III, Cambridge University Press, Cambridge, 1997.

Geweke, J., M. Keane and D. Runkle, "Alternative Computational Approaches to Inference in the Multinomial Probit Model," Review of Economics and Statistics, 76, 1994, pp. 609-632.

Geweke, J., M. Keane and D. Runkle, "Statistical Inference in the Multinomial Multiperiod Probit Model," Journal of Econometrics, 81, 1, 1997, pp. 125-166.

Gourieroux, C. and A. Monfort, Simulation Based Econometrics, Oxford University Press, New York, 1996.

Greene, W., "A Statistical Model for Credit Scoring," Stern School of Business, Dept. of Economics, Working paper \# 92-10, 1992.

Greene, W., "Gender Economics Courses in Liberal Arts Colleges: Further Results," Journal of Economic Education, fall, 1998, pp. 291-300.

Greene, W., "Fixed and Random Effects in Nonlinear Models," Working Paper 01-10, Department of Economics, Stern School of Business, Economics, 2001.

Greene, W., The Behavior of the Fixed Effects Estimator in Nonlinear Models, Stern School of Business, New York University, Department of Economics, Working Paper 02-03, 2002

Greene, W., Econometric Analysis, $5^{\text {th }}$ ed., Prentice Hall, Englewood Cliffs, 2003. (forthcoming)

Guilkey, D., and J. Murphy, "Estimation and Testing in the Random Effects Probit Model," Journal of Econometrics, 59, 1993, pp. 301-317.

Hajivassiliou, V., D. McFadden and P. Ruud, "Simulation of Multivariate Normal Orthatn Probabilities: Methods and Programs," Journal of Eeconometrics, 72, 1996, pp. 85-134. 
Hajivassiliou, V. and P. Ruud, "Classical Estimation Methods for LDV Models Using Simulation," In Engle, R. and D. McFadden, eds., Handbook of Econometrics, Vol. IV, North Holland, Amsterdam, 1994.

Hausman, J. and W. Taylor, "Panel Data and Unobservable Individual Effects," Econometrica, 49, 1981, pp. 1377-1398.

Heckman, J. 1981. The incidental parameters problem and the problem of initial conditions in estimating a discrete time-discrete data stochastic process. In Structural Analysis of Discrete Data with Econometric Applications, Manski, C. and McFadden D. (eds). MIT Press: Cambridge.

Lancaster, T., "The incidental parameters problem since 1948," Journal of Econometrics, 95, 2000, pp. 391-414.

L'Ecuyer, P, "Good Parameters and Implementations for Combined Multiple Recursive Random Number Generators," Department of Information Science, University of Montreal, working paper, 1998.

McFadden, D. and K. Train, "Mixed MNL Models for Discrete Response," Journal of Applied Econometrics, 15, 2000, pp. 447-470.

Morokoff, W., and R. Calflisch, "Quasi-Monte Carlo Integration," Journal of Computational Physics, 122, 1995, pp. 218-230.

Munkin, M. and P. Trivedi, "Econometric Analysis of a Self Selection Model with Multiple Outcomes Using Simulation-Based Estimation: An Application to the Demand for Healthcare," Manuscript, Department of Economics, Indiana University, 2000.

Nagin, D. and K. Land, "Age, Criminal Careers, and Population Heterogeneity: Specification and Estimation of a Nonparametric, Mixed Poisson Model," Criminology, 31, 3, 1993, pp. 327-362.

Ripley, B., Stochastic Simulation, John Wiley and Sons, New York, 1987.

Rubinfeld, D., "Voting in a Local School Election: A Micro Analysis," Review of Economics and Statistics, 59, 1977, 30-42.

Sepanski, J., "On a Random Coefficient Probit Model," Communications in Statistics - Theory and Methods, 29, 11, 2000, pp. 2493-2505.

Sloan, J. and H. Wozniakowski, "When are Quasi-Monte Carlo Algorithms Efficient for High Dimensional Integrals," Journal of Complexity, 14, 1998, pp. 1-33.

Stata Corp, Stata Statistical Software: Release 6.0, College Station, TX, Stata Corp., 1998.

Train, K., "Halton Sequences for Mixed Logit," Manuscript, Department of Economics, University of California, Berkeley, 1999.

Train, K., Discrete Choice Methods with Simulation, Cambridge University Press, Cambridge, 2002.

Wang, P., I. Cockburn, and M. Puterman, "Analysis of Patent Data - A Mixed Poisson Regression Model Approach," Journal of Business and Economic Statistics, 16, 1, 1998, pp. 27-41.

Wedel, M., W. DeSarbo, J. Bult, and V. Ramaswamy, "A Latent Class Poisson Regression Model for Heterogeneous Count Data," Journal of Applied Econometrics, 8, 1993, pp. 397-411.

White, H., "Maximum Likelihood Estimation of Misspecified Models," Econometrica, 50, 1982, pp. 1-25.

Woolridge, J., "Selection Corrections for Panel Data Models Under Conditional Mean Independence Assumptions," Journal of Econometrics, 68, 1995, pp. 115-132. 